Computer Applications: An International Journal (CAIJ), Vol.5, No.1, February 2018

\title{
DiGITAL EMOTION: How Audiences ReACT TO Robots ON SCREEN
}

\author{
Damian Schofield \\ Department of Computer Science, State University of New York, Oswego, NY, USA
}

\begin{abstract}
The experience of interacting with robots is becoming a more pervasive part of our day-to-day life. When considering the experience of interacting with other technologies and artefacts, interaction with robots presents a distinct and potentially unique component: physical connection. Robots share our physical space; this is a prominent part of the interaction experience. Robots offer a lifelike presence and the Human-Robot Interaction (HRI) issues go beyond the traditional interactions of more passive technologies and artefacts. The attention paid to HRI has grown dramatically as robotic systems have become more capable and as human contact with those systems has become more commonplace [1].
\end{abstract}

Immediately recognizable, culturally ubiquitous, androids, cyborgs, and robots, need no introduction. Yet their very familiarity obscures their participation in culture and media, and our perennial fascination with such artificial humans when seen on the screen. While robots are growing more capable of many tasks, people are often hesitant at introducing technology into older, more traditional art forms. However, robots of varying kinds are appearing with increasing frequency in all manner of cinematic productions [2].

Robots and artificial humans have been a staple of our sci-fi screen experiences, however, unlike previous technology such as smartphones or laptops, robots are currently being given more character roles in films. Therefore, like animated characters, audiences are beginning to anthropomorphize and have emotional experiences with the robot characters.

This paper attempts to unpack how humans see these artificial humans and how we interpret their representation in cinema through a discussion of the use of 'physical' robots as a natural next stage of cinema performance and drama. The paper presents and experiment involving cyborg performances in a series of short films. In this study, participants attended a screening where they viewed these films, and their responses to, and feelings about, the films were measured. It was hypothesized that film audiences have become comfortable with seeing robots in sci-fi films over the years. Therefore, it is expected that current and future audiences will begin to give these robot characters human attributes such as gender.

\section{KEYWORDS}

Robot, Human Robot Interaction, Cinema, representation, Appearance

\section{INTRODUCTION}

An artificial consciousness permeates globalized societies; technology is all around us, in science, in science fiction, in daily life. This relationship continues to be processual, technologies continue to move forward, assisting or, perhaps, encroaching on the human body. In modern society, we are increasingly becoming merged with the technology around us, wearing it and implanting it. This allows us to contemplate the merging of the organic and the inorganic. Bodies are being remapped by technology and rigid notions of subjectivity are reconfigured and societal norms are disrupted and shifted. Questions and issues regarding ability, identity, and a struggle for embedded agency in relation to technologies are principal concerns of the late twentieth and early 
Computer Applications: An International Journal (CAIJ), Vol.5, No.1, February 2018

twenty-first centuries [3]. Humans are bombarded by visual media and one often feels as if there is a fundamental invasion of body integrity [4].

This paper discusses media which has been designed to push the boundaries of what is traditionally described as film; providing a sterile environment where machines perform on a screen, robots reciting lines. Film is often defined as a form of literature which incorporates acting and stagecraft elements combined with a narrative script. The effectiveness of the film medium (which when conducted may be considered a play or drama, according to actual type) is based on the delivery of text through the actors and how the audience observing the performance responds. The introduction of robot thespians has the potential to create a form of cyborg cinema that challenges and re-examines the 'sensually different atmosphere' of cinema that we are used to [5].

Cinema is often described as a cultural construct and the 'liveness' and 'realism' debate is well documented $[6,7,8,9]$. Introducing robots as actors can be seen as removing the human agency which in turn can undermine the idea that performance is a specifically human activity and it may cast into doubt the existential significance attributed to performance. Auslander [10,11] claims that the concept of the 'live' emerges only as a result of mediatization and 'live' is, in the contemporary moment of globalized technology, already to some extent mediatized.

Morse [12] makes a case for machine subjects (such as the television or computer screen) and the cyberized machine-human interactions that increasingly take on the 'I' and 'you' of subjective construction (we talk to the television, to our phones etc). Socially constructed and based on what she calls virtualities the embodied, intelligent machine emerges as a partner in discourse.

A number of commentators have also noted that there have been noticeable changes in the styles of acting seen in film in recent years. Many mention a move towards a more mechanic, flattened, and intentionally 'non-acting' style, as humans sit alongside the technological on the screen $[3,13]$. The introduction of new forms of technology into cinema has challenged many notions of existing theory and practice and form complex alternatives. The introduction of robot thespians also highlights the fixed notions of what being human means in our modern world relative to the embodied and pervasive technologies that surround us.

However one sees the use of technology in cinema, there is no doubt that the cinema of the late twentieth and into the twenty-first centuries has been shaped by cultural processes. As the representational, visible bodies on the screen merge into the technology, Phelan proposes a new 'inclusive representational framework' - suggesting that the technology may efface their 'representational visibility' but in the process they are re-marked as something new, entering a cyborg sensitivity [14].

The concept of robot cinema raises a number of questions regarding the representation of the human body on the screen, providing an innovative site for exploring and experimenting with these ideas. If robot cinema is to progress, and to be used to help understand the impact of technology on human bodies, then the complex relationships between physical spaces, human bodies and technology needs to be examined. Removing humans from a film perhaps moves us closer to an understanding of a post-human condition [15]. A new, radically inclusive notion of 'universal subject' becomes necessary and a new critical language and way of thinking about film and performance becomes necessary.

Technology itself, can call the materiality of the body into question. Human bodies are increasingly abstracted, abjected, objectified through distance, media, commodification and technology [3]. A number of academics and researchers have asked if we should we lament the loss of the organic body [16]. In reality, technology development is often led by technological 
Computer Applications: An International Journal (CAIJ), Vol.5, No.1, February 2018

determinism, which feels that human bodies can naturally co-exist with technology as long as humans remain in control.

This paper considers the relationship between $21^{\text {st }}$ century research in robotics and the fantasy of the ideal robot, as this fantasy was honed in fictions, plays, and films of the twentieth century. It can be seen that that new versions of the artificial person in science fiction literature and film cannot escape many of the representational patterns of older texts. Cinema containing artificial people often returns to the same tropes and plotlines decade after decade [17].

If cyborg cinema is to be created and to develop then the complex issues between physical spaces, human bodies and technology needs to be discussed. This paper sets out to:

- Help understand the impact of technology on human bodies, and examine the notion of what being human means in our modern world relative to the embodied and pervasive technologies that surround us.

- Discuss media which has been designed to push the boundaries of what is traditionally described as film.

- Consider the relationship between $21^{\text {st }}$ century research in robotics and the fantasy of the ideal robot, as this fantasy was honed in fictions, plays, and films of the twentieth century.

- Raise a number of questions regarding the representation of the human body on the screen and to rehash and rethink the cyborg stereotypes seen in cinema.

\section{Defining Artificial Humans, Robots and Cyborgs}

In this section, we briefly survey events and work that have made modern robot technology possible. Although most robot technology was primarily developed in the mid and late 20th century, it is important to note that the notion of robot-like behavior and its implications for humans have been around for centuries in religion, mythology, philosophy, and fiction [18].

There are reports of automata and mechanical creatures from ancient Egypt, Greece, and China. The Iliad refers to golden maids that behave like real people [19]. The idea of golem, an "artificial being of Hebrew folklore endowed with life" has been around for centuries [20,21]. Ancient Chinese legends and compilations mention robot-like creations, such as the story from the West Zhou Dynasty (1066BC-771BC) that describes how the craftsman Yanshi presented a humanoid. The creation looked and moved so much like a human that, when it winked at the concubines, it was necessary to dismantle it to prove that it was an artificial creation [22].

During the Tang Dynasty, a craftsman, Yang Wullian made a humanoid robot which resembled a monk. It could beg for alms with a copper cup, put it in place after collecting and even bow down to the person who gave alms to the robot. All these movements were mechanically actuated and were either in a fixed sequence or under manual control [23]. Similar robotic devices, such as a wooden ox and floating horse, were believed to have been invented by the Chinese strategist Zhuge Liang [18], and a famous Chinese carpenter was reported to have created a wooden/bamboo magpie that could stay aloft for up to three days [24].

In the $15^{\text {th }}$ century, Leonardo da Vinci drew up schematics for a mechanical robot knight. It consisted of a knight's armor, which was fitted with gears, wheels and pulleys. It was controlled using cables and pulleys. This robotic knight could lift its visor, sit or stand and could move its head. Using the plans of the robotic knight made by Leonardo da Vinci, robotist Mark Rosheim 
Computer Applications: An International Journal (CAIJ), Vol.5, No.1, February 2018

built a prototype of the knight. He further modified the design and made it more advanced by introducing the ability to walk [25].

Early robot implementations were remotely operated devices with minimal autonomy. In 1898, Nicola Tesla demonstrated a radio-controlled boat, which he described as incorporating "a borrowed mind." In fact, Tesla controlled the boat remotely. Tesla hypothesized, “. . . you see there the first of a race of robots, mechanical men which will do the laborious work of the human race." He even envisioned one or more operators simultaneously directing 50 or 100 vehicles [18].

In the $20^{\text {th }}$ century we entered the era of robotics. An early example includes the Naval Research Laboratory's "Electric Dog" robot from 1923. Robots were created for many different purposes in multiple industries, including attempts to remotely pilot bombers during World War II, the creation of remotely piloted vehicles, and mechanical creatures designed to give the appearance of life [26]. In 1940, the first humanoid robot named Elektro [27] was created by Westinghouse Electric Corporation. It could only move its arms and head, move around on a wheel in its base, and it could play recorded speech. It consisted of photoelectric eyes and could distinguish between red and green light [23].

Complementing the advances in robot mechanics, research in artificial intelligence has attempted to develop fully autonomous robots. The most commonly cited example of an early autonomous robot was Shakey, which was capable of navigating through a block world under carefully controlled lighting conditions at the glacially slow speed of approximately 2 meters per hour [28]. Many agree that these early works laid a foundation for much that goes on in robot hybrid control architectures today [29,30].

The real challenge in production of autonomous humanoid robot is not just the designing but also programming and developing human functionality. It is important to design a humanoid robot as closely as possible to the design characteristics of a human being. The robot should also be able to communicate easily with the others and also should be able to take decisions on its own. The design was a difficult part to execute, since the extra ordinary balancing capability of the human being was not an easy task to understand and imply on a humanoid robot [23].

In 1973, Wabot-1, the first humanoid robot which could walk on two legs, communicate with a human and transport objects was created by Waseda University [31]. Although it could walk on two legs, the robot could only walk on flat surfaces.

A further breakthrough in autonomous robot technology occurred in the mid-1980s with work in behavior-based robotics [32,33]. Indeed, it could be argued that this work is a foundation for many current robotic applications. Behavior-based robotics breaks with the monolithic senseplanact loop of a centralized system, and instead uses distributed sense-response loops to generate appropriate responses to external stimuli. The combination of these distributed responses produces "emergent" behavior that can produce very sophisticated responses that are robust to changes in the environment.

Robot behaviors initially focused on mobility, but more recent contributions seek to develop lifelike anthropomorphic behaviors [34], acceptable behaviors of household robots [35], and desirable behaviors for robots that follow, pass, or approach humans [36,37,38].

Robots have also factored in multiple works of fiction, such as the mechanical-like birds that were present in the 1933 poem Byzantium by W. B. Yeats [39]. Robots have always had a large presence in science fiction literature, most notably the works of Isaac Asimov [40]. Many state 
Computer Applications: An International Journal (CAIJ), Vol.5, No.1, February 2018

that Asimov's Laws of Robotics acted as forerunners to the first design guidelines for humanrobot interaction metaphors.

\subsection{Definitions}

The word "robot" originates from the Czechoslovakian word robota which means work [23]. "Robot" appears to have first been used in Karel Chapek's 1920's play Rossum's Universal Robots (the character was a servant robot, which resembled the structure of a human being), though this was by no means the earliest example of a human-like machine [41].

The term cyborg was first used in 1960 to describe human-machine interfaces (cybernetic organisms) which could adapt to new environments, specifically space travel [42]. These cyborgs were intended to taken care of tasks automatically and unconsciously, leaving their creators free to explore, to create, to think, and to feel. A summary of accepted definitions is given below [43].

Cyborg: An organism with synthetic hardware which interacts directly with the brain, and alters the way it functions.

Robot: $\quad$ A machine designed to perform a task. A digitally driven creature that can sense and move.

Android: A robot designed to mimic human behavior and/or appearance.

Bionic: Any organism which has mechanical or robotic hardware designed to augment or enhance the body.

Sentient: Responsive to or conscious of some impression and context; aware.

The words 'robot', 'android' and 'cyborg' permeate modern culture, demonstrating a need for a radical rethinking about human positioning in the world. Our human subjectivity, seen in relation here to the digital technologies that surround us, becomes a shifting, difficult concept. Some argue that we are already cyborgs and therefore there is no need to question the shift; that humans are slipping into the technology world, appearing only as projections as we are becoming fully immersed in the technology $[44,45]$.

McLuhan and Moos [46] describe how we often see technology as an extension of our bodies, perhaps a response to existential and spiritual uncertainties, as we try to leave our fallible mortal bodies behind. A range of modern technologies are able to reconfigure our bodies as "dynamic fields of action in need of regulation and control" [16]. The terms robot and cyborg can be viewed in both a literal and metaphoric sense, asking questions regarding what it means to have a body, to share a body, and what it means to lose physical control of your own body [3].

Artificial people may be mechanical, but they may also be engineered through chemical or biotechnological means, cloned, altered, or reconstructed. While such modes of production reference technological realities, actual artificial people are truly imaginary, creatures of fiction, the imagination, and the magic of representational media. And yet despite their unreality they seem to inform a host of cultural domains and debates, participating in a dense web of interactions between fiction and reality in contemporary culture [17].

\subsection{Applications}

There are millions of robots in day-to-day use all around the world, and the rate of take-up of these systems is increasing rapidly [47]. Over time, it has been the goal for creators and manufacturers to expand the definition of what a robot is; in other words, the tasks robots are able to perform are continually expanding with manufacturing, hospitals and space exploration seen as 
Computer Applications: An International Journal (CAIJ), Vol.5, No.1, February 2018

common areas of interest for robotics [48,49]. It is generally felt that robots have emerged into an era of 'weak' Artificial Intelligence (A.I.) where currently they can imitate humans without being independent [50]. Either through autonomous means, or extensive exhaustive programming, robots have the potential to better everyday life.

This is perhaps nowhere more evident than in the very successful application of unmanned underwater vehicles that have been used to explore the ocean's surface to find lost ships, explore underwater life, assist in underwater construction, and study geothermal activity [51]. The development of robust robot platforms and communications technologies for extreme environments has also been successfully used by NASA and other international space agencies. Space agencies have had several high profile robotic projects, designed with an eye toward safely exploring remote planets and moons. Examples include early successes of the Soviet Lunokhods [52] and NASA's more recent success of exploring the surface of Mars [53,54].

Another of the major fields where humanoid robots have brought significant help is medical. For example, statistics have shown an epidemic increase since 1960's in cases of Autistic Spectrum Disorders (ASD). In recent years, robots have been increasingly used in autism diagnosis and treatment [55]. Humanoid robots have also been used for the treatment for cerebral palsy disabilities present in children that cause impairment in movement and posture [56]. Socially Assistive Robotics (SAR) is an example of a high end technology that assists humans in rehabilitation treatment of $\mathrm{CP}$ and ASD. Using human like responses from humanoid robots it has been possible to develop motor skills in $\mathrm{CP}$ patients and to Improving social and imitation skills in autistic children $[55,56]$.

Robot technology continues to develop, ever moving in the direction of increasing autonomy. Robot developers are working toward building robots that can act on their own, independent of specific direction from users. This type of "smart technology", as it is sometimes called, has begun to make its way into the everyday life of humans (Bernstein and Crowley, 2008).

Robot technology developers have started developing physical robots that interact with humans in everyday settings. These robots are known as social robots. Social robots hold a variety of different functions, including aiding the elderly, acting as tour guides, and even tutoring $[57,58]$. The robots can also have emotional roles, acting as companions, allowing people to cope with negative states such as depression, loneliness, and disability [59]. The use of robots in these areas has begun to open up a whole range of other areas of human endeavor to mechanical devices, including challenging areas of the arts and humanities that were traditionally the exclusive domain of humans $[60,61]$.

There are many different examples of autonomous robots: mechanical (or physical) robots, and software agents (softbots) which are now an everyday part of our internet experience in cyberspace [62]. This paper primarily focuses on physical robots, particularly those aspects that involve human interaction and communication. The paper also particularly focuses on the potential for robothespians to entertain in the emerging medium of cyborg cinema.

\subsection{NAO Robots}

The NAO is a humanoid robot created by Aldebaran Robotics - it takes advantage of the fact that by simply looking human, it is more likely that human viewers will attribute the robot actors with more human-like qualities than machine-like qualities. A NAO robot (Figure 1) has 25 individual servo motors offering many degrees of freedom, which allows the robot to move in a similar manner to humans. The $57 \mathrm{~cm}$ tall robot has the ability to visually and verbally recognize humans, the dual cameras embedded in its eyes allow the robot to 'see' its surroundings. The NAO robot can also respond to a human by recognizing sounds using two audio microphone/speaker units, 
positioned on each side of the head and is capable of delivering speech using a variety of text-tospeech and recording mechanisms.

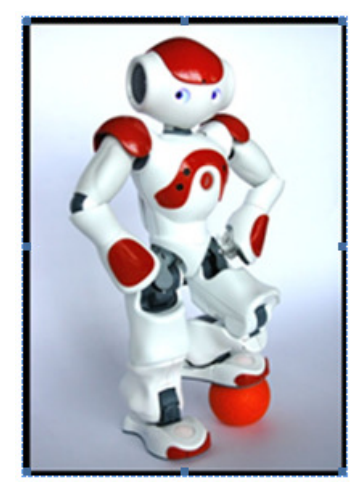

Figure 1: The Nao Robot

The NAO robots are unable to change their facial expression; they are also unable to independently change their gaze [63]. The NAO robot is able to use different LED colors in the eyes to depict changes in expression, but it is appreciated that this may not much of an emotional dynamic range.

Communication was seen as a critical component of this project to create cyborg cinema. Research has shown (perhaps via a naïve biology mechanism), that humans attribute verbal communication to robots if they have mouths. According to one study, the attribution of verbal communication is enough for people to start a conversation with the robot [58].

Experiments have indicated that the perception of robots can change if they act inappropriately and out of context [64]. NAO robots can be programmed to react in several types of manners. Since its degrees of freedom are similar to that of a human's, biomechanical modeling can mimic human emotional body language. Also, advanced audio controls can adjust the delivery tone of speech. The NAO robot has been used to a limited extent theatrically, they have been used in research projects around storytelling and comedy. Recently, the robots were used by French researchers to narrate stories to children. The researchers report that the NAO robots possesses the ability to carefully and closely mimic real life human behavior, and are capable of reproducing human-like gestures. Using 89 different story segments with different speech styles, an elaborate lexicon was produced [65].

A social roboticist named Heather Knight has presented a NAO robot as a comedian in a stand-up comedy show. The robot not only told jokes, but could actually pay attention to the audience and determine whether the audience was laughing and then adapt its comedy routine [66,67].

\section{RELATED WORK ON ROBOTS IN CINEMA}

Although theatre has been around for thousands of years, robots have inhabited the earth for only a couple of decades. However, there exists a long and rich history of technology being integrated with theatre, acting and performance dating back to the ancient Greeks. These have ranged from tools used in the mechanics of theatre (winches and revolves for example), the integration of complex props into performances, the use of realistic mannequins and puppets, to the use of technological themes within the narratives themselves. Historically, following Aristotle's elements of drama; theatrical forms that rely on technological effects are named as a 'spectacle', and are often considered as entertainment rather than serious drama (Laurel, 2013). 
Computer Applications: An International Journal (CAIJ), Vol.5, No.1, February 2018

There is a long history of film practitioners investigating and trying out computer technology; however the late $20^{\text {th }}$ century showed an increased amount of experimentation with technology. During this period, the rapid pace of technological development was reflected and mirrored in performance contexts in films all over the world [7]. This upsurge in multimedia performance demanded of scholars and reviewers a new critical language to accurately describe and analyse the work of this nature.

The majority of film productions utilizing digital technology have focused on computer generated special effects and characters $[69,70,71,72]$. Modern cinema creates multiple fantastic worlds and 'spectacles' that constantly clamor for our attention. However, the acceptance of Computer generated technology in film has not been universally positive and many push back against the changes $[73,74,75]$.

As modern consumers we all live tied to our own personal, ubiquitous, interactive digital devices. New technologies are developed and subsequently introduced and experimented with in media contexts. Artists and film pioneers continue to push the boundaries of old and new media in their efforts to explore the ongoing relationship between technology and human bodies. Traditionally, technologies have had a tendency to contain and limit bodies, fixing them on screen, as if viewed through lenses. In a cinema context, the appropriation of these technologies has sometimes reiterated or exposed these restraining boundaries [3].

Although there have been many examples of entertainment robotics, including the use of robots as robotic story tellers [76], robotic dance partners [77], robotic plants that give users information such as incoming email [78], and robotic pets [79,80]. However, from a research perspective, not much has been published in the literature. Early entertainment robotics centered on animatronics, where the robot generally plays prerecorded sounds that are synchronized with the robots motion. These types of robots can often be found in old movies and theme parks; however, the interaction is mostly in one direction, that of the robot presenting information, although the robot's performance may be triggered by the presence of the human. However, the 2005 AICHI Expo demonstrated several robots designed to entertain, including the use of robots as actors and dance partners [18]; similar work on the relationship between acting, drama, and artificial agents is presented in recent work using robots as improvisational performers. However, here again, the role of the human is as an observer, and the interaction is minimal and more implicit [81].

Recently, we have also started to see artificial 'physical' characters on theater stages, such as the one introduced in Richard Maxwell's play, Joe [82]. Although the robot does not literally merge or interact with other live bodies in this piece, the very introduction of such technology on stage introduces the concept of a whole new era of cyborg theatre and cinema. The first dedicated robotic theatre has recently opened at the Copernicus Science Centre in Warsaw, Poland [83]. Although this playhouse is relatively new, robotic acting has been occurring in other countries for many years. For example, in 2008, it was reported by BBC that Mitsubishi had created a robot named Wakamaru which spoke lines of script in Japanese. Wakamaru, a humanoid robot, performs in plays which emphasize the relationship between "humanity and technology" [84].

When human actors are replaced in cyborg cinema, is there a need to represent differing genders and races that remain distinct among human actors on the material stage or do we meld all the stereotypes together into a single cohesive entity represented by the robot actor ? Cyborg cinema is an extension of the tension and attention exhibited by the audience; it allows us to deeply investigate technology and the audience's reactions to it. Cyborg cinema also pushes the boundaries of 'posts' into new territories: post-private, post-identity, post species, post organic. $[3,85]$. 
Computer Applications: An International Journal (CAIJ), Vol.5, No.1, February 2018

Many feminist theorists have already extensively discussed the ideas proposed by cyborg cinema, particularly the way the technology challenges existing notions of subjectivity in our modern world $[4,14,64,86,87,88,89]$. Parker-Starbuck [3] claims that when considering cyborg subjectivity gender still matters, that individual bodies (human or robotic), however abled, raced, sexed, all matter in the formation of a subjectivity that opens out to encourage a composite position.

Ultimately, the robot actors are bodies on the screen. The cyborg actors form links and connections with the audience through technologies in a presumed cyborg consciousness [3]. These robot actors are often immersed within technology, but resist being absorbed by it; there is a smooth acceptance by most audiences that evades gender, sexuality, age, race, class, ability.

\section{Audience Reaction to Cyborg Cinema}

It has been stated that acting itself is a simulated activity, a false activity [90]. Cinema is also often seen as a transient activity that does not provide a commodifiable end product. The products from a cinema screening are intangible - even when the film provides satisfaction, excitement, passion or connectedness.

Filmic media can be classified beyond the limitations of language. Identity and identification are always based on projection, the audience collects images of the actors on the screen, watching and interacting with their stories, in relation to our notions of ourselves. The introduction of a range of new technologies promises new ways of looking at film and an elimination of the actoraudience divide, including novel modes of understanding immersive experiences. Performances involve many audiences and actors, forming bonds with and through technological environments and with techno-subjects [91].

It is provocative to imagine films that are made without the direct participation of live people [82]. Computer-generated characters have been used in films for many years, but there are still usually live actors on the screen with them. When we start to consider cyborg cinema and robot actors, the only 'live' component is the audience. Isherwood [15] claims that it is possible to create humane, affecting works of performance without the literal presence of human beings. The audience may understand the experience of cyborg cinema intellectually, but when physically confronted with a film with no human actors, perhaps this understanding needs to be at a different level. And even when the technologies are well integrated into the film and fairly transparent, the missing physical bodies can still be disquieting.

Human actors are often in close proximity to the technologies surrounding them on a film set and rely upon theatrical vocabulary to balance the experience for the audience. Humans on screen are delicately placed as equally weighted components with technologies in these settings, frequently creating seemingly seamless performances with the spectators in the movie theater.

Cyborg cinema is not aiming to replicate the traditional experience of cinema or film; instead the aim is to create a fusion of forms reliant on the embodied actor on screen, interacting with the audience through various technologies. The performance is being mediated electronically, the audience sees a network that joins them all [91,92]. Historically, the interplay between film and audience has produced a relationship that refuses to let human bodies fully disappear. There is a feedback loop between the actors and their projected counterparts, illustrating for the spectators the theatrical interplay with film techniques that at times focus the audience's eyes on the screen itself and at other times on the bodies on the screen (Gates, 2010). 
Computer Applications: An International Journal (CAIJ), Vol.5, No.1, February 2018

However, the introduction of other more advanced technologies onto the screen, cyborg cinema in particular has the potential to create large, more intricate feedback loops. Cyborg cinema challenges our modes of viewing and engaging with the performance, making us aware of disparities between human and robot performers. Audiences may be confused as to how they are suppose to approach intricate collections of sampled sound, digital images, and moving machine parts that make up a cyborg cinema performance $[4,94]$.

This struggle between media and bodies, where to look and for how long, frames a tension in the audience. As more technology is introduced onto the screen there is always the possibility that audiences will remain detached, almost clinical observers, as the performance is revealed through a process of mediation. Alternatively the introduction of technology onto the screen can be regarded as introducing a new, interesting form that could perhaps extend cinema's life, and from this practical perspective facilitate a future of movie goers for generations addicted to their mobile phones, computer games and other screens $[3,95]$.

Usually when a robot is seen on screen there's little to no data as to how the audience perceives the presence of the robot in the film. Yet, each film that incorporates an artificial character helps in maintaining and promoting the idea of advanced technology in culture [96]. The NAO robot used in the experiment described in this paper is a humanoid robot and therefore can be seen by audiences as having a more human appearance/representation.

Glaskin [97] believes the everyday social interaction the public now has with advanced A.I. technology allows people to begin to anthropomorphize robots, which, in turn, will then allow for acceptance. Oyedele et al [98] proposed in their study that

"It is expected that both the context within which humans and robots interact and the extent to which the robot resembles a human will influence affect toward the robot".

\section{EXPERIMENTAL METHOD}

The purpose of this research experiment is to analyze audience response to NAO robots, portrayed in a series of short films. The research is is concerned with how the fears or anxieties audiences have toward artificial humans can influence their level of comfort when watching these films. It is hypothesized that watching past sci-fi films, have influenced audiences' comfortability as they watch robots perform in the films used in this experiment. It is predicted that older audiences will be less comfortable with the presence of A.I. in film. It is also expected that participants would be more likely to assign a gender to artificial human characters from films with human characters performing alongside robot characters. This experiment aims to provide statistical data on the anthropomorphizing of artificial humans in film.

The film making process was an interdisciplinary experience involving media students from RMIT University in Melbourne, Australia working with Human-Computer Interaction (HCI) students from the State University of New York (at Oswego), USA. Scripts for short films that focused on robot technology were written by the Australian students. The American students filmed the scripts in the USA. The robots used were the autonomous, programmable and humanoid NAO robots [96].

The three short films produced had very different plots :

- "Robbot" is about a woman who's husband died and she decided to replace him with a robotic husband (Figure 2a).

- "Jonno and Mate" is about an man and a robot becoming friends (Figure 2b). 
- The third film is a re-interpretation of a scene from the book, "Do Androids Dream of Electric Sheep" [97].

This collaborative experience involved an interdisciplinary team with multiple skills: writing, acting, directing, programming, filming, editing, post-production. The production involved multiple participants interacting with the robots in many different ways.

The robot film making experience allowed the research team to investigate HRI as a behavioral process, where the filmmakers and actors interact with physical robots and the audience interact with robot representations displayed on a screen. Each role in the production had specific expectations associated with it; particularly with respect to the robots performing a form of 'social' interaction with the cast and crew members. For example, the writers needed to know about the robots properties, their capabilities and their potential behavior in each scene. For acting with robots, the actors/actresses needed to know what they can expect from their co-performers (the robots). In this respect, not only the design of the physical properties, or robot appearance, is important, but also the design of its behavior is a very important issue [98].

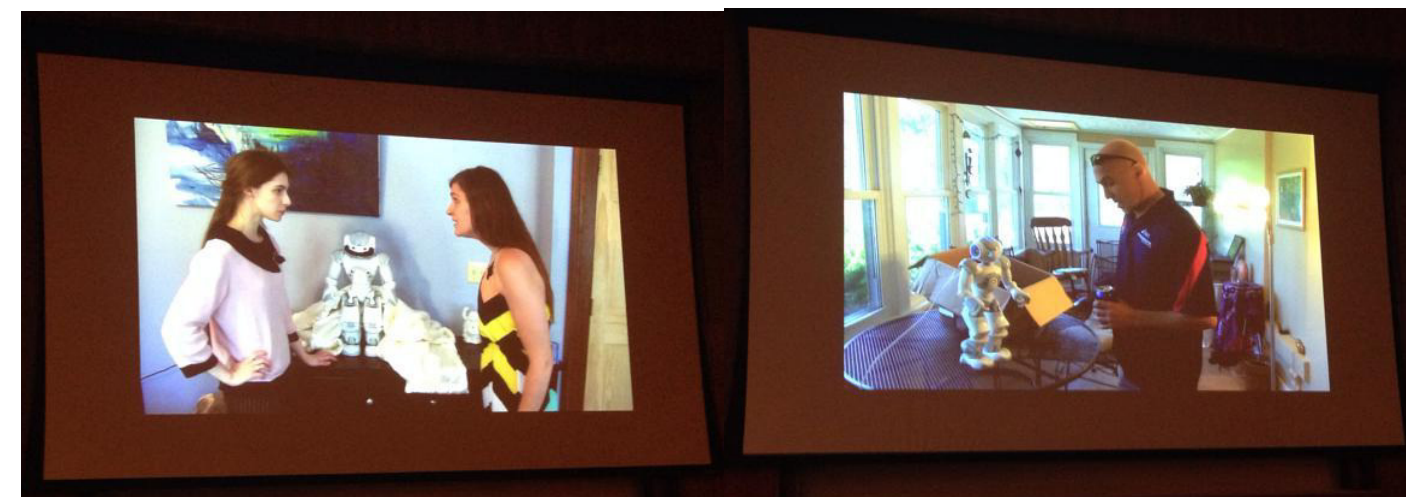

Figure 2a: The Robbot Movie

Figure 2b: The Jonno and Mate Movie

Looking at interactions through this lens of cinema gives a number of ways to solve some major HRI problems. To overcome the articulation problem, we must learn how human actors move, in a way that can be translated to the limited degrees of freedom on a robot. Using skilled actors as a model presents a way to get a limited amount of motion to be subtly nuanced, intended to convey the actor's intent.

In general, bringing in cinema professionals (actors, directors, choreographers, etc.) to provide a feedback loop about how they see a robot's motions should also be useful, as they are trained in a way that roboticists generally are not to view movement with a more precise eye. They can use that knowledge to overcome the robot's mechanical constraints and help overcome the intentionality problem.

Further, by realizing the movement's position in a larger dramatic arc, treating an interaction like a performance piece can help disambiguate a robot's motions. While a particular movement can be ambiguous, consciously putting it in a larger context can help make it specific and clear. Also, sensing becomes slightly easier by using labelled human motions performed by actors as a way to help interpret what the humans are doing, solving one small portion of the interpretation problem. Additionally, controlled performative experiences can often eliminate the need for a precise sensing metric. Finally, we can use the judgments of film criticism and untrained audience reactions to help evaluate how well the robots perform [5]. 
The development of a robot performance is dependent on accuracy; the robots need to be in exact positions on the set to allow props to be passed between them. Therefore, the timing of movement and delivery of the lines was deemed to be a key feature underlying the programming the robot performance. To ensure that every line was said in sequence and none were skipped, the lines were programmed as a series. In other words, when one line ended, the next would begin. The next line could not be delivered until the last line had been finished. Individual chunks of programming code were created, and these were linked together to form a timed movement flowchart, where all of the robot actor's cue were set, so that individual sections of the code would run in the correct order.

Figure 3 shows a small section of the programming flowchart controlling one of the robots, each icon in the figure represents a section of programming code. To give some idea of the complexity of the task involved in programming the robot thespians, it should be noted that this section of the flowchart controls no more than a few seconds of the performance of one robot. In the initial performance the text to speech capabilities of the NAO robots was used to generate the audio for each of the lines delivered during the performed scene. Lines were timed with a stopwatch to validate that lines by the robot weren't being spoken too slow or too fast.A number of dress rehearsals of the scene were performed in the robot laboratory. When the cyborg performers were ready, the robots were filmed performing their roles in the film scenes on stages and sets in the university theatre department's acting laboratory.

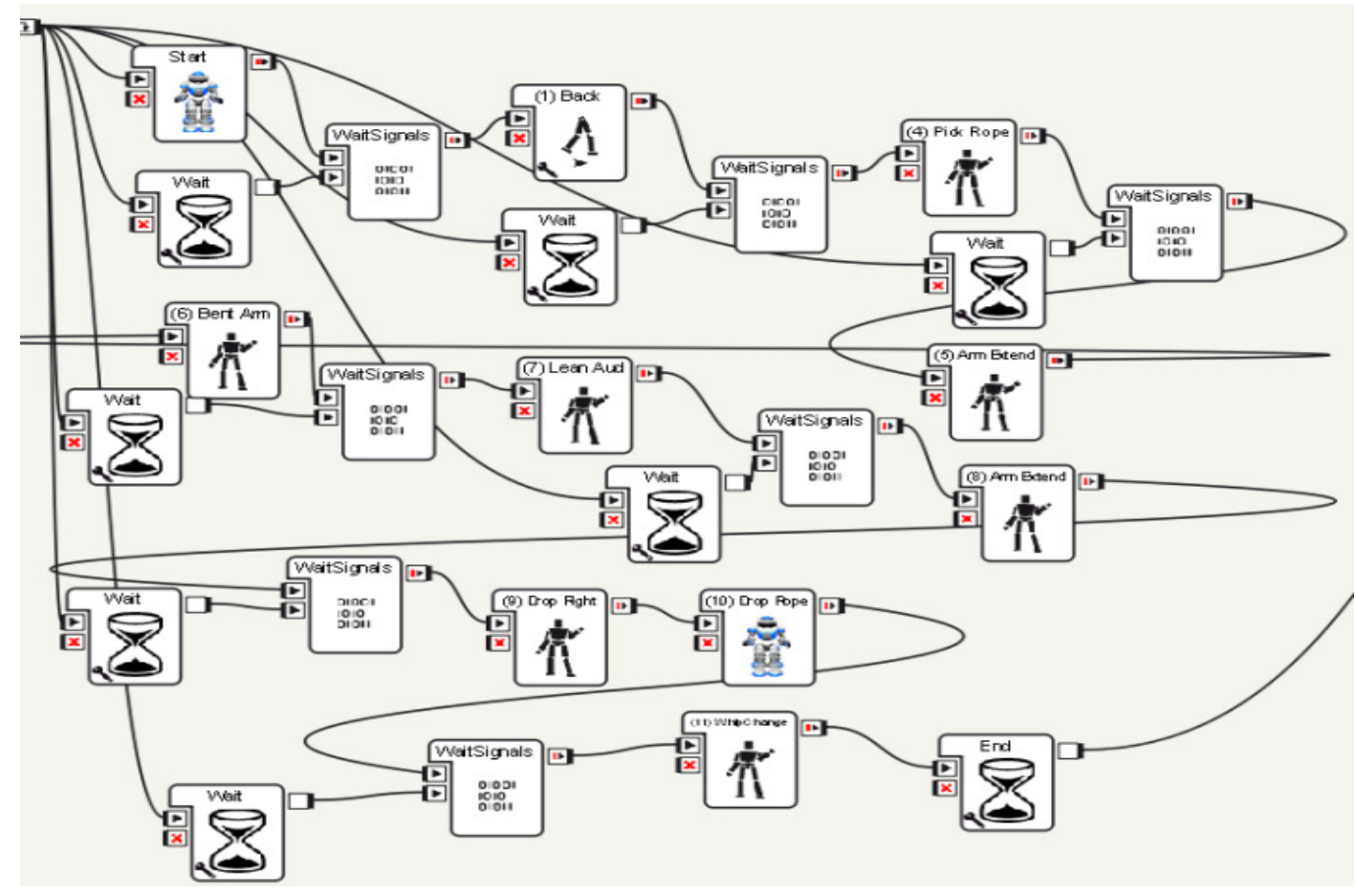

Figure 3: A Portion of the Robot Control Flowchart Linking Individual Sections of Code

Participants were shown three films which incorporated artificial humans as actors. Two of the films shown had a NAO robot playing a major role in the cast. After viewing the films, participants were then asked to complete a brief questionnaire.

The transhumanism questionnaire allowed for participants to record their responses to multiple questions about the films that were seen on a 10-point scale. The purpose of the questionnaire was to measure how the participants reacted to the films. One portion of the questionnaire was to 
decide how participants would view artificial humans in films in the future. Another portion of the questionnaire was used to decide how participants felt about the current film. Questions were asked on the participants' fear or feelings towards the robots in the film as well as how well they felt they understood the films' robots. The next section of the questionnaire was concerned with whether or not the participants associated the robots with a specific gender.

After data was collected, participant data was placed into separate groups based on their age group. Participants aged 18-21 were seen as Group A. Participants aged 22-25 were seen as Group B. Participants aged 26-29 Group C, 30-33 Group D and 54-58 Group E.

\section{RESULTS}

For this study, age and gender are seen as independent variables with comfort, emotions (fear and weirdness) and future perceptions are dependent variables. There was a total of 81 participants used in this study $(\mathrm{N}=81)$, a larger participant pool than many other comparable experiments in the literature. A single factor ANOVA test was conducted to compare the effect of age on comfort, fear, weirdness and future perception. The results were not found statistically significant, $\mathrm{F}(5,468)=45.22, \mathrm{p}=4.47$.

Group A ( $\mathrm{n}=44, \mathrm{M}=4.08, \mathrm{SD}=1.15)$, Group B $(\mathrm{n}=29, \mathrm{M}=4.10, \mathrm{SD}=1.11)$ and Group C $(\mathrm{n}=$ $4, \mathrm{M}=4.75, \mathrm{SD}=.05)$ responded as being comfortable when watching the NAO robots in the film. Group D ( $=1, M=5, S D=0)$ and Group $\mathrm{E}(\mathrm{n}=2, \mathrm{M}=5, \mathrm{SD}=0)$ responded as being very comfortable when watching the Nao robots in the film. In total all the groups were comfortable while watching the Nao robots in the films $(M=4.15, \mathrm{SD}=1.11)$. However, the groups with the older aged participants responded as being very comfortable watching the robots in the film versus the groups with the younger participants. (See Figure 4) This can be due to older audiences having the opportunity to watch more A.I. films than younger audiences.

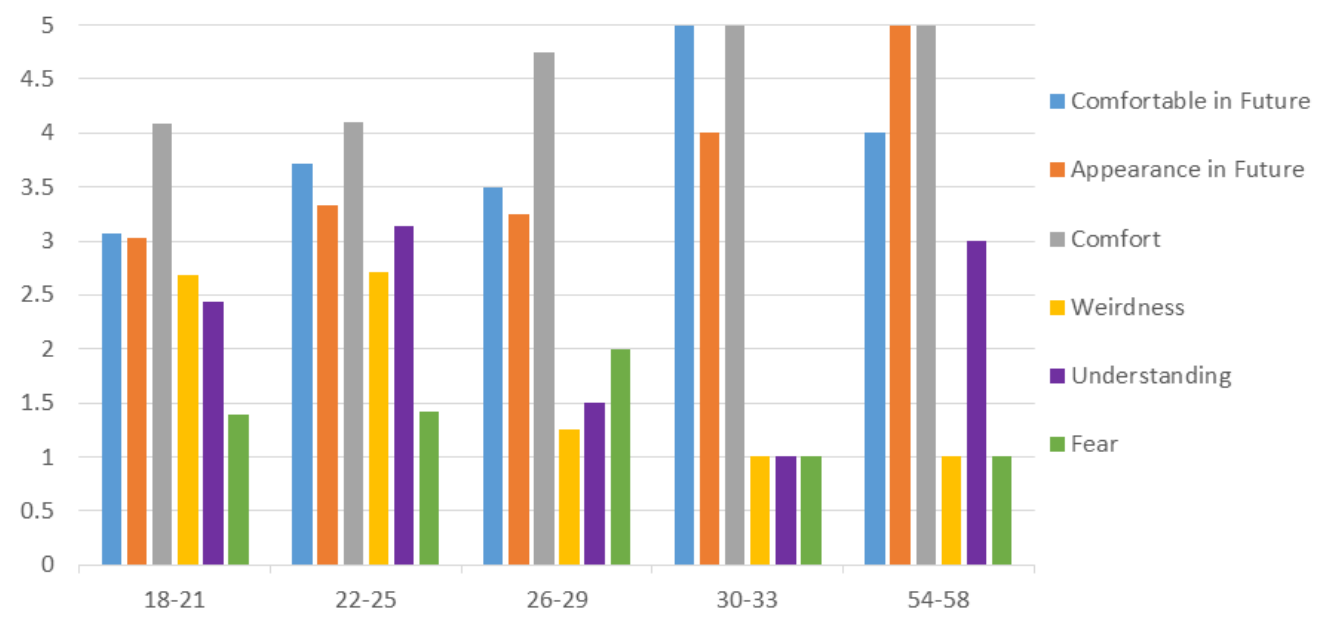

Figure 4. Averages of Transhumanism Questionnaire Responses

Group A (M=1.39, SD = 0.69), B (M=1.41, SD = 0.82), $\mathrm{D}(\mathrm{M}=1, \mathrm{SD}=0)$ and $\mathrm{E}(\mathrm{M}=1, \mathrm{SD}=0)$ were not scared at all when watching the robots being in the film. Group $\mathrm{C}(\mathrm{M}=2, \mathrm{SD}=2)$ was moderately scared when watching the robots in the film. Group $\mathrm{C}(\mathrm{M}=1.5, \mathrm{SD}=1)$ and $\mathrm{D}(\mathrm{M}=1$, $\mathrm{SD}=0)$ thought that it was easy to understand the robots. Group $\mathrm{A}(\mathrm{M}=2.44, \mathrm{SD}=1.24)$ found the robots understandable. Group $\mathrm{B}(\mathrm{M}=3.13, \mathrm{SD}=1.32)$ and $\mathrm{E}(\mathrm{M}=3, \mathrm{SD}=2.82)$ were unsure about how much they could understand from the robots. Group A $(\mathrm{M}=2.68, \mathrm{SD}=1.30)$ and Group B $(\mathrm{M}=2.70, \mathrm{SD}=1.42)$ found the robots to be slightly weird while watching the film. 
Computer Applications: An International Journal (CAIJ), Vol.5, No.1, February 2018

However, Group $\mathrm{C}(\mathrm{M}=1.25, \mathrm{SD}=0.50), \mathrm{D}(\mathrm{M}=1, \mathrm{SD}=0)$ and $\mathrm{E}(\mathrm{M}=1, \mathrm{SD}=0)$ did not find the robots weird at all. So, while the participant in Group E was not scared of the robots in the film, they were still unsure with how much they understood from the robots but still did not find the robots weird. Group A on the other hand were not scared at all and found the robots understandable and still slightly weird.

Group A $(M=3.02, S D=1.37), B(M=3.33, S D=1.44)$ and $C(M=3.25, S D=2.06)$ thought that audiences in the future could possibly be watching virtual actors and robot actors. Group $D(M=4$, $\mathrm{SD}=0$ ) thought that it was very possible that future audiences will watch virtual actors and robot actors in the future. Group $\mathrm{E}(\mathrm{M}=5, \mathrm{SD}=0)$ was definitely sure that audiences would watch virtual actors and robot actors in the future. Group A $(\mathrm{M}=3.07, \mathrm{SD}=1.17), \mathrm{B}(\mathrm{M}=3.72, \mathrm{SD}=$ $1.22)$ and $\mathrm{C}(\mathrm{M}=3.50, \mathrm{SD}=1.91)$ are somewhat comfortable with the idea of watching virtual actors and robot actors in the future. Group $\mathrm{E}(\mathrm{M}=4, \mathrm{SD}=1.41)$ was comfortable with the idea of watching virtual actors and robots in the future. Group $\mathrm{D}(\mathrm{M}=5, \mathrm{SD}=0)$ was very comfortable with the idea of watching virtual actors and robots in the future. Once again, Groups E and D are more comfortable with watching A.I. in films in the future than younger groups.

Participants were asked their thoughts on the inclusion of gender roles within the films. While viewing the Robbot film, all of the participants thought that there were some gender roles given to the robot in the film $(\mathrm{M}=3.73, \mathrm{SD}=1.36)$. For the Johnno film, both female $(\mathrm{n}=44, \mathrm{M}=2.30$, $\mathrm{SD}=1.42)$ and male $(\mathrm{n}=33, \mathrm{M}=2, \mathrm{SD}=1.29)$ participants found the robot in the film to not have a prominent gender role. In the Do Androids film, male $(\mathrm{M}=2.33, \mathrm{SD}=1.15)$ found the robots in the film to not have prominent gender roles. While female $(\mathrm{M}=1.57, \mathrm{SD}=0.97)$ participants found the robots in the film to have a gender roles. Out of the three films, participants found the Robbot film's artificial human to have the highest level of gender $(\mathrm{M}=3.73, \mathrm{SD}=1.36)$.

\section{ANALYSIS}

During the production of the robot films, a number of technical aspects were deemed worthy of assessment. The productions were deemed a success, and the films were considered ready for public viewing, when all of the following criteria were met.

- Firstly, the length of the scenes involving the robots should be approximately the same length as scenes with human actors. The length of the production was deemed important because this will indicate how successful the robots were at moving into position and making correct movements.

- Secondly, the NAO robots must deliver all the lines from the film scripts correctly, at the appropriate times, without missing any or making mistakes.

- Thirdly, the cyborg thespians must also complete the passing, grabbing, and picking up of props successfully. The success of props and lines will be a pass/fail condition, since these usually work in succession

- Lastly, once the programing and preparation is completed, the robots should be able to perform the scenes in one complete sequence. The overall performance of the scene can be viewed (in a technical capacity) as a pass/fail for every time both robots are run. After weeks of preparation (comparable to rehearsal time) the robot thespians were ready to be filmed.

There was a general feeling among the audience members that robots still need to improve in regard to movement. It was felt that the NAO robots did not possess as many degrees of freedoms as humans. Some movements were deemed too difficult for the robot to perform on camera - for example, the ability to rotate the lower body. Walking is a very common movement, but the NAO 
Computer Applications: An International Journal (CAIJ), Vol.5, No.1, February 2018

robots walk differently than humans. It is very obvious in the robot films that this affected the experience of the audience who noticed these unnatural movements. Grasping is also difficult to perform, as the human hand is very complex and is inherently difficult to replicate. For example, the NAO robots only have 3 fingers. One acts in opposition from the other two. Joints in the fingers cannot act independently in these robots, this created problems in the nuanced manipulation of some of the props.

The physical appearance of the cyborg thespians on screen was not seen as a problem by the audience. In the feedback, the audience were very accepting of the robot actors. It was felt that part of this was due to their engagement with the innovative nature of the robot performance. Interestingly, a number of audience members stated that they saw the robots as genderless.Probably the biggest issue for robots while performing in the films is the lack of consciousness. Robots don't know where the director wants them to start a scene. They won't automatically figure out where they need to walk to, or altering their movement direction perhaps in response to something changing on set. If robots were truly autonomous, with strong A.I., they would behave more like true human actors, altering and improvising as the situation dictates. A sense of consciousness however is not the same as volition. That is, being able to learn and to improve, is not equivalent to a free will. It is arguable that many actions and lines produced are dictated so by a director or writer. Thus, one could argue that actors themselves do not possess volition. They merely follow a set of choreographed movements and repeat memorised lines.

Along with the development of robotic interfaces, there has been more research published on the evaluation of these systems. HRI researchers have employed a variety of evaluation styles in their work; they can evaluate their systems summatively (i.e.,after-the-fact) or formatively (i.e., during system development). The problem with applying heuristic evaluation to HRI, however, is the validity of using existing usability and efficiency heuristics for HRI. In the past, critics have often evaluated the performance of actors through the prism of how well they connected with the audience. A similar evaluation was used to help define and refine an evaluation method of robots and how well they connected with their audience. In this work HRI is not only evaluated in a cinematic setting, but also a measure of how well the human cast and audience react and connect to the robots in these settings.

Two of the films in the study strongly featured NAO robots as characters within the film (Robbot and Johnno). The third film used human actors to play the role of the artificial human (Do Androids). In Robbot, the Nao robot in the film played the character of a woman's husband. The woman programmed the robot to respond to situations as her husband would. So, she designed the robot in the image of her deceased husband. In the film Johnno, a robot was programmed to help a professor with his work. However, the robot ended up in the hands of a man other than the professor. The audience responses in this study demonstrated that artificial characters are usually seen as separate from the human race. Instead, these characters are part of their own race of robots.

Based on the premises of the films, it was interesting to find that participants saw the Do Android characters to have no gender roles. Especially since the actors in the film were male and female. The fact that participants thought that the films with actual robots as actors showed stronger gender roles is somewhat perplexing. However, it could suggest that the participants of the study were familiar with artificial gender roles from past films. Also, the film Robbot specifically showed that the robot in the film was there to replace a character's husband.

In certain cultures, the use of humanoid robots is more prominent than others. Glaskin [99] explains how in Japanese culture, there is more exposure to humanoid robots than in other cultures. In American culture, the idea of robots artificial humans is widely displayed in film culture. There is an assumption that the technology demonstrated in films will become available 
Computer Applications: An International Journal (CAIJ), Vol.5, No.1, February 2018

to consumers in the future. Therefore, one can conclude that robot technology in films encourages the future production of technology and vice versa.

\section{Conclusions}

In conclusion, the NAO robots were able to perform in a series of short films successfully. A number of issues were identified by the audience members, mainly relating to movement of the robots. Of course, the larger research question, even in a simple experiment such as this, is the efficacy of using robots as actors to perform in films such as this and their effect upon an audience. It is apparent that when an audience compares cyborg and human cinema, they will probably compare what the robotic actors lack to (what they believe are) perfected, human actors. Therefore, this comparison is biased since currently robots still lack autonomy, human motion and advanced language processing. However, cyborg thespians offer a degree of control and precision not available in human actors. As in the human cinema, the success of the cyborg cinema will primarily depend on the response of the audience.

The amount of robot actors in films will only continue to grow. Since the characters in most films are reflexive of human traits and behaviors, humans can relate to these characters. By relating with these characters, audiences are also anthropomorphizing them. This is a fundamental framework for the idea of transhumanism to continue to develop in audiences. In return, audiences will also be able to give these characters' human traits such as gender. This allows for the idea of the presence of artificial humans. to become more normalized in society. The data analyzed in this study demonstrated, that the more audiences view robots in film, the more comfortable they will be with future robots playing roles in films. This is supported by older participants being more comfortable with artificial humans in future films than younger participants. More research is needed to support the findings in this paper. However, this study does provide a strong argument for the reason why audiences can relate to A.I. in films.

This paper has outlined the ways in which cyborg cinema involving robot thespians has already provided a deeper understanding of how intentional or coincidental robot actions might impact human perception. While it is acknowledged that cinematic contexts are often distinct from natural sociability, robotic interaction schemas generally place humans at the center of overall task goals, thus there are many overlapping lessons we can glean from the construct of an actor and audience.

Future work should continue to evaluate cross-applications of social knowledge from dramaturgical theory to robot behavior systems, and envision contexts for cyborg cinema that frame the audience as a user study full of participants.

\section{REFERENCES}

[1] Clarkson, E., and Arkin, R.D. (2007) "Applying Heuristic Evaluation to Human-Robot Interaction Systems", Proceedings of FLAIRS Conference, Florida, USA, pp 44-49.

[2] Lu, D.V. (2012) "Relative Comfort of People with Acting Robots by Theatre and Technology Experience", TinyToCS, 1.

[3] Parker-Starbuck, J. (2011) Cyborg Theatre: Corporeal / Technological Intersections in MultimediaPerformance, Basinstoke, UK: Palgrave Macmillan.

[4] Braidotti, R. (2002) Metamorphoses: Towards a Materialist Theory of Becoming, Cambridge, UK: Polity Press.

[5] Glasser, J. (1955) "Forms of Modern Drama”, Comparative Literature, 7(2), 129-143..

[6] Barthes, R. (1977) “The Grain of the Voice”, In S. Heath (Trans), Image, Music, Text, New York: Hill and Wang, pp 179-189.

[7] Dixon, S. (2007), Digital Performance: A History of New Media in Theatre, Dance, Performance Art, and Installation, Cambridge, MA: MIT Press. 
Computer Applications: An International Journal (CAIJ), Vol.5, No.1, February 2018

[8] Glesekam, G. (2007) Staging the Screen: The Use of Film and Video in Theatre, Basingstoke, UK:Palgrave Macmillan.

[9] Reinelt, J.G. and Roach, J.R. (2007) "Mediatized Cultures", In J.G. Reinelt and J.R. Roach (Eds), Critical Theory and Performance, Ann Arbor: University of Michigan Press, pp 521-525.

[10] Auslander, P. (1997) From Acting to Performance: Essays in Modernism and Postmodernism, London, UK: Routledge.

[11] Auslander, P. (1999) Liveness: Performance in a Mediated Culture, London, UK: Routledge.

[12] Morse, M. (1998) Virtualities: Television, Media Art and Cyberculture, Bloomington: Indiana University Press.

[13] Bay-Cheng, S. (2007) "Theatre Squared: Theatre History in the Age of Media”, Theatre Topics, 17(1), pp 37-50.

[14] Phelan, P. (1993) Unmarked: The Politics of Performance, London, UK: Routledge.

[15] Isherwood, C. (2010) "Detailed Reflections, Verbal and Visual", New York Times, 14th January 2010.

[16] Cartwright, L. (1995) Screening the Body: Tracing Medicine's Visual Culture, Minneapolis: University of Minnesota Press.

[17] Kakoudaki, D. (2014) Anatomy of a Robot: Literature, Cinema, and the Cultural Work of Artificial People, New Jersey: Rutgers University Press.

[18] Goodrich, M.A. and Shultz, A.C. (2007) "Human-Robot Interaction: A Survey", Foundations and Trends in Human Computer Interaction, 1(3), pp 203-275.

[19] Homer, (circa 800 BC) The Iliad. Book XVIII.

[20] Webster, (1996) Webster's Seventh New Collegiate Dictionary, Springfield, MA: G. and C. Merriam Company.

[21] Wiener, N., (1966) God and Golem Inc.: A Comment on Certain Points where Cybernetics Impinges on Religion, Cambridge, MA, USA: MIT Press.

[22] Youkou, L., (circa 500 BC) Book of Master Lie.

[23] Gizem, Aksahya \& Ayese, Ozcan (2009) Coomunications \& Networks, Network Books, ABC Publishers.

[24] Tzu, M. (circa 400 BC) Book of Master Mo.

[25] Rosheim, M.E., (2006) Leonardo’s Lost Robot, Berlin: Springer Verlag.

[26] Fong, T. and Thorpe, C., (2001) "Vehicle Teleoperation Interfaces", Autonomous Robots, 11(1), pp 918.

[27]Televox, H., (1983) "The Robots of Westinghouse", History of Computers, Retrieved From: http://historycomputer.com/Dreamers/Elektro.html.

[28] Bainbridge, L., (1983) "Ironies of Automation", Automatica, 19(6), pp 775-779.

[29] Murphy, R.R., (2000) Introduction to AI Robotics, Cambridge, MA: The MIT Press.

[30]Parker, L.E., (1994) "ALLIANCE: An Architecture for Fault Tolerant, Cooperative Control of Heterogeneous Mobile Robots", Proceedings of IEEE/RSJ/GI International Conference on Intelligent Robots and Systems, Munich, Germany, 776-783.

[31] Takahashi, T., (2006) Robotto no tensai [He is the Genius Makes a Robot (sic)], Tokyo: Media Factory Inc./ Robo Garage.

[32] Arkin, R.C., (1998) Behavior-Based Robotics, Cambridge, MA: The MIT Press.

[33]Brooks, R.A., (1986) “A Robust Layered Control System for a Mobile Robot", IEEE Journal of Robotics and Automation, 2, pp 14-23.

[34] Yamaoka, F., Kanda, T., Ishiguro, H., and Hagita, N., (2005) "Lifelike Behavior of Communication Robots Based on Developmental Psychology Findings", Proceedings of IEEERAS International Conference on Humanoid Robots, Tsukuba, Japan.

[35] Kim, H.J., Lee, H., and Kim, C., (2007) "Autonomous Behavior Design for Robotic Appliance", Proceedings of ACM/IEEE International Conference on Human-Robot Interaction, Arlington, VA, USA

[36] Gockley, R., Forlizzi, J., and Simmons, R., (2007) "Natural Person-Following Behavior for Social Robots", Proceedings of ACM/IEEE International Conference on Human-Robot Interaction, Arlington, VA, USA.

[37] Pacchierotti, E., Christensen, H.I., and Jensfelt, P., (2006) "Evaluation of Passing Distance for Social Robots", Proceedings of IEEE International Workshop on Robot and Human Interactive Communication, Hatfield, UK.

[38] Walters, M.L., Dautenhahn, K., Woods, S.N., and Koay, K.1., (2007) "Robot Etiquette: Results from User Studies Involving a Fetch and Carry Task", Proceedings of ACM/IEEE International Conference on Human-Robot Interaction, Arlington, VA, USA. 
Computer Applications: An International Journal (CAIJ), Vol.5, No.1, February 2018

[39] Yeats, W.B., (1933) The Winding Stair and Other Poems, Reprinted by Kessinger Publishing.

[40] Asimov, I., (1986) Robot Dreams, New York: Ace Books..

[41] Capek, K., and Capek, J., (1961) R.U.R. and The Insect Play, London: Oxford University Press..

[42]Clynes, M.E., and Kline, N.S., (1995) "Cyborgs and Space", in C.H. Grey (Ed), The Cyborg Handbook, New York: Routledge, pp 29-33.

[43]Garreau, J., (2005) Radical Evolution: The Promise and Peril of Enhancing our Minds, Our Bodies And What It Means To Be Human, New York: Doubleday..

[44] Caygill, H., (1997) "Stelarc and the Chimera", Art Journal, 56(1), pp 46-57.

[45] MacKenzie, J., (2001) Perform or Else: From Discipline to Performance, New York: Routledge.

[46]McLuhan, M., and Moos, M.A., (1997) Media Research: Technology, Art, Communication, Amsterdam: G \& B Arts..

[47] IFR, (2016) Industrial Robot Statistics - 2016, International Federation of Robotics.

[48] Presher, A., (2010) "Robotics Make a Move Toward Autonomous Service", Design News, 65(6), pp $\mathrm{m} 12-\mathrm{m} 14$.

[49]Behnke, S., (2008) “Humanoid Robots - From Fiction to Reality?” KL-zeitschrift, 4(8), pp 5-9.

[50]Beck, A., Cañamero, L., and Bard,K.A., (2010) "Towards an Affect Space for Robots to Display Emotional Body Language". Proceedings of 19th International Symposium on Robot and Human Interactive Communication, Viareggio, Italy.

[51]Whitcomb, L.L., (2000) "Underwater robotics: Out of the Research Laboratory and into the Field", Proceedings of IEEE International Conference on Robotics and Automation, San Francisco, CA.

[52]Fong, T., Thorpe C., and Baur, C., (1999) "A Robot-Centric Model for Vehicle Teleoperation", Proceedings of AAAI Spring Symposium: Agents with Adjsutable Autonomy, Stanford, CA.

[53]Leger, P.C.,Trebi-Ollennu, A., Wright, J.R., Maxwell, S.A., Bonitz, R.G., Biesiadecki, J.J., Hartman, F.R., Cooper, B.K., Baumgartner, E.T., and Maimone, M.W., (2005) "Mars Exploration Rover Surface Operations: Driving Spirit at Gusev Crater", Proceedings of IEEE International Conference on Systems, Man, and Cybernetics, Hawaii

[54]Wilcox B., and Nguyen, T., (1998) "Sojourner on Mars and Lessons Learned for Future Planetary Rovers", Proceedings of SAE International Conference on Environmental Systems, Danvers, MA, USA.

[55]Taheri, A.R., Alemi, M., Meghdari, A., Pour Etemad, A.H., and Holderrea, S.L., (2015) "Clinical Application of Humanoid Robots in Playing Imitation Games for Autistic Children in Iran", Procedia Social and Behavioral Sciences, 176, pp 898-906.

[56]Rahman, R.A., Hanapiah, F.A., Basri, H.H., Malik, N.A., and Yussof, H., (2015) "Use of Robot in Children with Cerebral Palsy: Ups and downs in Clinical Experience”, Procedia Computer Science, 76, pp 394-399.

[57]Bernstein, D., and Crowley, K., (2008) "Searching for Signs of Intelligent Life: An Investigation of Young Children's Beliefs about Robot Intelligence", Journal of the Learning Sciences, 17, pp 225-247.

[58]Fior, M., Ramirez-Serrano, A., Beran, T., Nugent, S., and Kuzyk, R., (2010) "Children's Relationships with Robots: Robot is Child's New Friend", Journal of Physical Agents, 4(3), pp 9-17.

[59]Libin, A., and Libin, E., (2004) "Person-Robot Interactions from the Robopsychologists' Point of View: The Robotic Psychology and Robotherapy Approach”, Proceedings of the IEEE, 92(11), pp 1789 - 1803.

[60]Hatano, G., Siegler, R.S., Richards, D.D., Inagaki, K., Stavy, R., and Wax, N., (1993) "The Development of Biological Knowledge: A Multi-National Study”, Cognitive Development, 8, pp 4762.

[61]Barakova, E., and Lourens, T., (2010) "Expressing and Interpreting Emotional Movements in Social Games with Robots", Personal Ubiquitous Computing, 14, pp 457-467.

[62]Zhao, S., (2006) "Humanoid Social Robots as a Medium of Communication", New Media and Society, 8(3), pp 401-419.

[63]Niemüller, T., Ferrein, A., Eckel, G., Pirro, D., Podbregar, P., Kellner, T., Rath, C. and Steinbauer, G., (2011) "Providing Ground-Truth Data for the Nao Robot Platform", Lecture Notes in Computer Science, 6556, pp133-144..

[64]Goetz, J., Kiesler, S. and Powers, A., (2003) "Matching Robot Appearance and Behavior to Tasks to Improve Human-Robot Cooperation", Proceedings of the 12th IEEE Workshop on Robot and Human Interactive Communication (RO-MAN).

[65]Gelin, R., d'Alessandro, C., Le, Q.A., Deroo, O., Doukhan, D., Martin, J., Pelachaud, C., Rilliard, A. and Rosset, S., (2010) "Towards a Storytelling Humanoid Robot", Proceedings of the International Dialog with Robots Conference - Advancement in Artificial Intelligence (AAAI).. 
Computer Applications: An International Journal (CAIJ), Vol.5, No.1, February 2018

[66]Knight, H., (2011), "Eight Lessons learned about Non-verbal Interactions through Robot Theater", Social Robotics, Springer Berlin Heidelberg, pp42-51.

[67]Knight, H, Veloso, M. and Simmons, R., (2015) "Taking Candy from a Robot: Speed Features and Candy Accessibility Predict Human Response". Proceedings of International Conference on Robot and Human Interactive Communication (Ro-Man '15).

[68]Laurel, B., (2013) Computers as Theatre, New York: Pearson Education..

[69]Youngblood, G., and Fuller, R.B., (1970) Expanded Cinema, New York: Dutton.

[70]Manovich, L., (2001) The Language of New Media, Cambridge, MA: MIT press.

[71]Ohta, Y., and Tamura, H., (2014) Mixed Reality: Merging Real and Virtual Worlds, New York: Springer.

[72]Schofield, D., (2016) “The Use of Computer Generated Imagery in Legal Proceedings", Digital Evidence \& Electronic Signature, 13(3).

[73]Catmull, E., (1978) “The Problems of Computer Animation”, ACM SIGGRAPH Computer Graphics, 12(3), pp 348-353.

[74]Magnenat-Thalmann, N., and Thalmann, D., (1987) "The Direction of Synthetic Actors in the Film Rendezvous à Montréal”, IEEE Computer Graphics and Applications, 7(12), pp 9-19.

[75]Clark, D., (2014) Computers for Imagemaking, London: Elsevier.

[76]Montemayor, J., Alborizi, H., Druin, A., Hendler, J., Pollack, D., Porteous, J., Sherman, L., Afework, A., Best, J., Hammer, J., Kruskal, A., and Lal, A., (2000) "From PETS to Storykit: Creating New Technology with an Intergenerational Design Team”, Proceedings of Workshop on Interactive Robots and Entertainment (WIRE), Pittsburgh, PA.

[77]Kosuge, K., Hayashi, T., Hirata, Y., and Tobiyama, R., (2003) "Dance Partner Robots - Ms. DancerR", Proceedings of IEEE/RSH International Conference on Intelligent Robots and Systems, Las Vegas, NV.

[78]Jacobs, P., (2003) "When is a Garden . . . Not a Garden?" Landscape Architecture, 10.

[79]Fong, T., Nourbakhsh, I., and Dautenhahn, K., (2003) "A Survey of Socially Interactive Robots", Robotics and Autonomous Systems, 42, pp 143-166.

[80]Shibata, T., Tashima, T., and Tanie, K., (1999) "Emergence of Emotional Behavior Through Physical Interaction Between Human and Robot", Proceedings of IEEE International Conference on Robotics and Automation, Detroit, Michigan.

[81]Bruce, A., Knight, J., Listopad, S., Magerko, B., and Nourbakhsh, I., (2000) "Robot Improv: Using Drama to Create Believable Agents", ICRA.

[82]Maxwell, R., (2002) Joe, PS122, New York:MaxBooks.

[83]Poynton, M., (2016) "Science Fiction Theatre as an Aspect of Digital Participatory Culture", (Doctoral Dissertation). State University of New York at Buffalo, USA.

[84]Ogawa, K., Chikaraishi, T., Yoshikawa, Y., Nishiguchi, S., Hirata, O., and Ishiguro, H., (2014) "Designing Robot Behavior in Conversations Based on Contemporary Colloquial Theatre Theory", Robot and Human Interactive Communication, pp 168-173..

[85]Schofield, D., and Young, D., (2016) "Waiting for Robot Godot: A Cyborg Theatre Case Study", International Journal of Contemporary Humanities, 1(1).

[86] Albright, A.C., (1997) Choreographing Difference: the Body and Identity in Contemporary Dance, Middletown: Wesleyan University Press.

[87] Haraway, D.J., (1991) Simians, Cyborgs, and Women: The Reinvention of Nature, New York: Routledge.

[88] Hayles, N.K., (1999) How we Became Post-Human, New York: Cornell University Press.

[89] Thompson, R.G., (1997) Extraordinary Bodies: Figuring Physical Disability in American Culture and Literature, New York: Colombia University Press.

[90] Dias, P., Freedmn, A., Medway, P. and Pare, A., (2013) Worlds Apart: Acting and Writing in Academic Workplace Contexts, Routledge.

[91] Kaye, N., (2007) Multimedia: Video-Installation-Performance, Routledge, London..

[92] Farman, J., (2009) "Surveillance Spectacles: The Big Art Group's Flicker and the Screened Body in Performance", Contemporary Theatre Review, 19(2), pp181-194.

[93] Gates, A., (2010) “The Screen's Now Setting Many a Stage”, New York Times, 2nd March.

[94] Helfand, G., (1996) "Electronic Eye", Bay Guardian, 27th March.

[95] Clough, P.T., (2007) Introduction. In The Affective Turn: Theorizing the Social, Durham, Duke University Press.

[96] Aldebaran Robotics, (2013) NAO, Corporate Website, available from : http://www.aldebaranrobotics.com/en/ 
Computer Applications: An International Journal (CAIJ), Vol.5, No.1, February 2018

[97] Dick, P.K., (1968), Do Androids Dream of Electric Sheep, New York: Ballantine Books.

[98] Hiroi, Y., and Ito, A., (2011) "Toward Human-Robot Interaction Design through Human-Human Interaction Experiment”, in D. Yang (Ed.), Informatics in Control, Automation and Robotics, 2(133), Berlin: Springer, pp 127-130.

[99] Glaskin, K., (2012) "Empathy and the Robot: A Neuroanthropological Analysis", Annals of Anthropological Practice, 36, pp 68-87.

\section{Author}

Damian Schofield is currently Director of Human Computer Interaction (Full Professor) at the State University of New York (SUNY) at Oswego, a position he has held since November 2009. He also currently Adjunct Associate Professor of Forensic Computing at Edith Cowan University, Perth, Western Australia.

Prior to his move to America, Dr. Schofield held the position of the Associate Professor of Computer Games and Digital Media, in the School of Creative

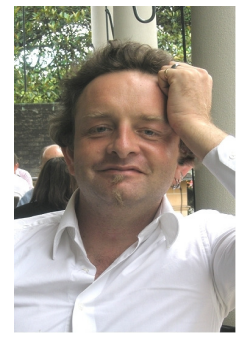
Media at RMIT University in Melbourne, Australia. Before his move out to Australia, he was Lecturer in the School of Computer Science at the University of Nottingham, UK. Dr. Schofield also remains a director and major shareholder of Aims Solutions Ltd., a UK company, that provides computer graphics visualization services and virtual reality based simulation training products to a wide range of organizations.

Dr. Schofield has been involved in research examining the use of digital media and technology in a variety of commercial environments for over 20 years. He is particularly interested in virtual reconstructions (using computer games/graphics technology) and the introduction of robot and A.I. technology in new areas. 